\title{
Gas-cushioned Droplet Impacts with a Thin Layer of Porous Media
}

\author{
Peter D. Hicks · Richard Purvis
}

Received: date / Accepted: date

\begin{abstract}
The pre-impact gas cushioning behaviour of a droplet approaching touchdown onto a thin layer of porous substrate is investigated. Although the model is applicable to droplet impacts with any porous substrate of limited height, a thin layer of porous medium is used as an idealized approximation of a regular array of pillars, which are frequently used to produced superhydrophobic and superhydrophilic textured surface. Bubble entrainment is predicted across a range of permeabilities and substrate heights, as a result of a gas pressure build-up in the viscous-gas squeeze film decelerating the droplet free-surface immediately below the centre of the droplet. For a droplet of water of radius $1 \mathrm{~mm}$ and impact approach speed $0.5 \mathrm{~m} \mathrm{~s}^{-1}$ the change from a flat rigid impermeable plate to a porous substrate of height $5 \mu \mathrm{m}$ and permeability $2.5 \mu \mathrm{m}^{2}$ reduces the initial horizontal extent of the trapped air pocket by $48 \%$, as the porous substrate provides additional pathways through which the gas can escape. Further increases in either the substrate permeability or substrate height can entirely eliminate the formation of a trapped gas pocket in the initial touchdown phase, with the droplet then initially hitting the top surface of the porous media at a single point.

Droplet impacts with a porous substrate are qualitatively compared to droplet impacts with a rough impermeable surface, which provides a second approximation for a textured surface. This indicates that only small pillars can be successfully modelled by the porous media approximation. The effect of surface tension on gas-cushioned droplet impacts with porous substrates is also investigated. In contrast to the numerical predictions of a droplet free-surface above flat plate, when a porous substrate is included the droplet free-surface touches down in finite time. Mathematically this is due to the regularization of the parabolic degeneracy associated with the small gas-film-height limit the gas squeeze film equation, by non-zero substrate permeability and height, and physically suggests that the level of surface roughness is a critical parameter in determining the initial touchdown characteristics.
\end{abstract}

P. D. Hicks

School of Engineering, Fraser Noble Building, King's College, University of Aberdeen, Aberdeen, AB24 3UE, United Kingdom.

Tel.: +44 (0)1224 274815

E-mail: p.hicks@abdn.ac.uk

R. Purvis

School of Mathematics, University of East Anglia, Norwich Research Park, Norwich, NR4 7TJ, United Kingdom.

E-mail: r.purvis@uea.ac.uk 
Keywords Droplet impacts · porous media - gas entrainment

\section{Introduction}

Droplet impacts with porous or textured surfaces form an integral part of many industrial processes, not least in ink-jet printing [6]. Whilst microfibre mats [23] and micro-structured surfaces $[40,41,32]$ are increasingly being designed to control surface wetting properties by inducing hydrophobic or hydrophilic effects, droplet impacts onto such surfaces have only recently received attention $[36,13]$. These droplet impact studies demonstrate that porous substrates can dramatically reduce splashing and related behaviour. Similar differences have been observed in impacts onto capillary tubes [7] and onto anisotropic substrates [9]. Improved insight into the physical mechanisms at play is vital to better understanding and improved design and, whilst there is an increasing amount of experimental research, analytical progress is still limited.

Recent studies of supercooled droplet impacts onto micro-structured surfaces [26,27], motivated by developing non-wettable surfaces to prevent aircraft icing, observed an unexpected feature; significant pre-impact droplet distortion caused by air-cushioning. This manifests itself in visualisation of the wetted area of the effectively porous substrate, with trapped air bubbles clearly visible. Such bubble entrapment has been detailed and measured for solid substrates for many years, see for example the bubble measurements and visualisations in $[4,37,38]$, the investigations into the air and liquid dynamics detailed in $[10,43$, $39,25,33$ ] and closely related work in droplet coalescence [8]. However these recent experiments with micro-structured surfaces were the first time similar phenomena have been found for a porous substrate.

Possibly due to the lack of experimental evidence, there has been little analytical study of small-scale impacts with porous surfaces. Similar phenomena have been studied in larger scale impacts however, for example [5] considers jetting in wave impact onto a permeable barrier, and [18] considers self-similar solutions for the water entry of a porous wedge. However, in contrast with the current concern which is in part viscosity dominated, these previous studies have assumed purely inviscid flow, in line with the larger length scales and impact speeds present there. For smaller droplet impact, viscosity is of vital importance in the narrowing air-layer between the approaching droplet and porous substrate as impact is approached.

Building on earlier work $[35,31,15]$, we consider an asymptotic model of air-cushioning of droplets impacting upon a porous substrate, as an initial attempt to understand this observed behaviour. Given the relatively small length scales, we establish that a suitable model couples inviscid flow in the water droplet, with viscous lubrication flow in the air and with Darcy flow in the substrate. While there has been previous studies combining porous media with squeeze film flow, notably in models of the nip press used in paper manufacture [12] and in a mathematical description of knee cartilage [21], the complex porous-viscous-inviscid coupling described here is rather novel.

\section{Model Development}

An idealized two-dimensional liquid droplet of radius $R$, approaches impact with a porous substrate of height $\tilde{y}=\tilde{h}$ at a normal impact velocity $U$. A sketch of this problem is shown in 


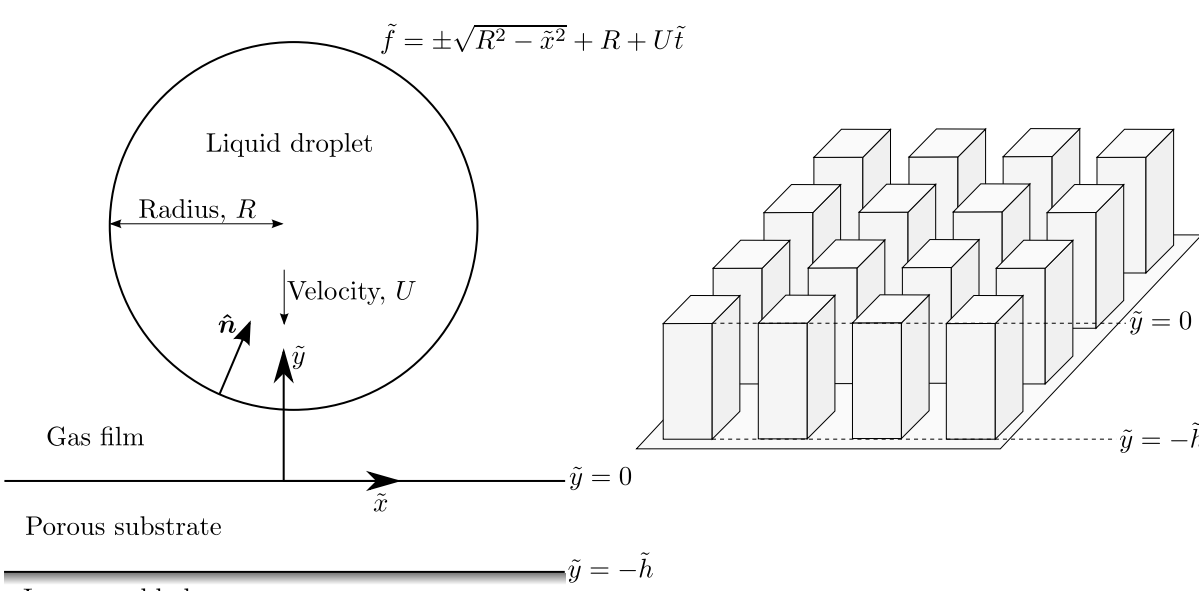

Impermeable base

Fig. 1: A sketch of a liquid droplet approaching touchdown with a porous layer (left) and an idealized sample array of rectangular pillars as might be found on a textured superhydrophobic surface (right).

figure 1. Away from impact, the undisturbed droplet free-surface $\tilde{f}$, at horizontal position $\tilde{x}$ and time $\tilde{t}$, is given by

$$
\tilde{f}= \pm \sqrt{R^{2}-\tilde{x}^{2}}+R+U \tilde{t}
$$

The liquid in the droplet is assumed to be incompressible and therefore the liquid velocities $\tilde{\boldsymbol{u}}_{l}=\left(\tilde{u}_{l}, \tilde{v}_{l}\right)$ in the $\tilde{x}$-direction parallel to the substrate interface and the $\tilde{y}$-direction perpendicular to the substrate interface are governed by the Navier-Stokes equations in the form

$$
\tilde{\nabla} \cdot \tilde{\boldsymbol{u}}_{l}=0, \quad \frac{\partial \tilde{\boldsymbol{u}}_{l}}{\partial \tilde{t}}+\tilde{\boldsymbol{u}}_{l} \cdot \tilde{\nabla} \tilde{\boldsymbol{u}}_{l}=-\frac{1}{\rho_{l}} \tilde{\nabla} \tilde{p}_{l}+\frac{\mu_{l}}{\rho_{l}} \tilde{\nabla}^{2} \tilde{\boldsymbol{u}}_{l} .
$$

Here a subscript $l$ denotes a property of the liquid, $\tilde{p}_{l}$ is the liquid pressure, $\rho_{l}$ is the liquid density, $\mu_{l}$ is the dynamic viscosity of the liquid and $\tilde{\nabla}=\left(\frac{\partial}{\partial \tilde{x}}, \frac{\partial}{\partial \tilde{y}}\right)$.

If the ambient gas pressure $p_{0}$ is such that

$$
U \ll\left(\frac{p_{0}^{3} \mu_{g}}{\rho_{l}^{4} R}\right)^{1 / 7},
$$

where $\mu_{g}$ is the dynamic viscosity of the gas, then the gas is also incompressible to leading order $[28,16]$ and is governed by the incompressible Navier-Stokes equations

$$
\tilde{\nabla} \cdot \tilde{\boldsymbol{u}}_{g}=0, \quad \frac{\partial \tilde{\boldsymbol{u}}_{g}}{\partial \tilde{t}}+\tilde{\boldsymbol{u}}_{g} \cdot \tilde{\nabla} \tilde{\boldsymbol{u}}_{g}=-\frac{1}{\rho_{g}} \tilde{\nabla} \tilde{p}_{g}+\frac{\mu_{g}}{\rho_{g}} \tilde{\nabla}^{2} \tilde{\boldsymbol{u}}_{g}
$$

where a subscript $g$ denotes a property of the gas phase, while the meaning of the other variables matches that in the liquid droplet. For a water droplet of radius $R=1 \mathrm{~mm}$ in air at atmospheric pressure, condition (3) gives a maximum impact velocity for incompressible gas behaviour $U \ll 1.53 \mathrm{~m} \mathrm{~s}^{-1}$. If the impact velocity approaches or moderately exceeds this value, then gas compressibility effects are significant at leading order and a modified 
viscous lubrication equation and coupled energy conservation equation are required [17]. Given the typically low impact speeds considered here, the relatively moderate changes that compressibility induces (see [17] or [25]), and the reduced gas pressures found for porous substrates, compressibility is neglected in the current study.

It shall subsequently be shown that the gas film behaviour is dominated by viscous effects. In the substrate gas flows more slowly than in the gas film and consequently inertial effects can be neglected here also. Assuming the porous medium to be isotropic, then incompressible Darcy flow in the substrate satisfies

$$
\tilde{\nabla} \cdot \tilde{\boldsymbol{u}}_{s}=0, \quad \text { and } \quad \tilde{\boldsymbol{u}}_{s}=-\frac{K}{\mu_{g}} \tilde{\nabla} \tilde{p}_{s},
$$

where a subscript $s$ denotes a property of the gas in the pore spaces of the substrate or of the substrate itself.

At the interface between the liquid droplet and the gas film $\tilde{y}=\tilde{f}(\tilde{x}, \tilde{t})$, the kinematic boundary condition and the normal stress balance, imply

$$
\begin{aligned}
\rho_{h}\left(\tilde{\boldsymbol{u}}_{g}-\tilde{\boldsymbol{u}}_{i}\right) \cdot \hat{\boldsymbol{n}} & =\rho_{l}\left(\tilde{\boldsymbol{u}}_{l}-\tilde{\boldsymbol{u}}_{i}\right) \cdot \hat{\boldsymbol{n}} \\
\hat{\boldsymbol{n}} \cdot\left(\tilde{\mathbb{T}}_{g}-\tilde{\mathbb{T}}_{l}\right) \cdot \hat{\boldsymbol{n}} & =\frac{\tilde{\sigma} \tilde{f}_{\tilde{x} \tilde{x}}}{\left(1+\tilde{f}_{\tilde{x}}^{2}\right)^{3 / 2}},
\end{aligned}
$$

where $\tilde{\boldsymbol{u}}_{i}$ is the velocity of the interface, $\hat{\boldsymbol{n}}$ is a unit normal vector pointing into the droplet, $\tilde{\sigma}$ is the surface tension coefficient on the liquid-gas interface and the stress tensor $\tilde{\mathbb{T}}=-\tilde{p} \mathbb{I}+$ $\mu\left[\tilde{\nabla} \tilde{\boldsymbol{u}}+(\tilde{\nabla} \tilde{\boldsymbol{u}})^{T}\right]$.

At $\tilde{y}=0$, the interface between the gas film and the porous substrate, the normal stress balance and the continuity of normal velocity implies

$$
-\tilde{p}_{g}+2 \mu_{g} \frac{\partial \tilde{v}_{g}}{\partial \tilde{y}}=-\tilde{p}_{s}, \quad \text { and } \quad \tilde{v}_{g}=\tilde{v}_{l},
$$

respectively. The Beavers-Joseph condition [2]

$$
\frac{K^{1 / 2}}{\alpha} \frac{\partial \tilde{u}_{g}}{\partial \tilde{y}}=\tilde{u}_{g}-\delta \tilde{u}_{s}
$$

allows a velocity slip on the interface between the gas and the porous bed. Here $\alpha$ is the Beavers-Joseph coefficient (which depends on the material and pore properties in the boundary region), and the parameter $\delta=1$ if slip is allowed, while $\delta=0$ corresponds to no slip. The terms $\tilde{u}_{g}$ and $\partial \tilde{u}_{g} / \partial \tilde{y}$ involving the gas velocity are evaluated at $\tilde{y}=0+$, while there is a thin layer just below the plane $\tilde{y}=0$ over which the velocity transitions to the substrate velocity $\tilde{u}_{s}[30]$. At height $\tilde{y}=-\tilde{h}$, the base of the porous substrate, a no penetration condition $\tilde{v}_{s}=0$ is applied.

\subsection{Droplet behaviour}

The droplet behaviour close to the point of touchdown can be determined in the nondimensional coordinate system

$$
(x, y, t)=\left(\frac{\tilde{x}}{\varepsilon R}, \frac{\tilde{y}}{\varepsilon R}, \frac{U \tilde{t}}{\varepsilon^{2} R}\right),
$$


where the time scale is taken to be the time required for the droplet to cross a narrow gas film of height $\varepsilon^{2} R$ assuming no deformation occurs and $\varepsilon$ is a small parameter whose value will be determined shortly. Liquid velocities are scaled by the impact velocity $U$, which gives dimensionless velocities, pressure and free-surface position

$$
(u, v, p, f)=\left(\frac{\tilde{u}}{U}, \frac{\tilde{v}}{U}, \frac{\varepsilon \tilde{p}}{\rho_{l} U^{2}}, \frac{\tilde{f}}{\varepsilon^{2} R}\right) .
$$

Here the pressure is scaled to retain its presence at leading order, while the droplet freesurface is scaled by the height of the narrow gas film which separates the lower droplet free-surface from the porous substrate.

For $\varepsilon \ll 1$ and $U \gg \mu_{l} / \rho_{l} R$, the convective acceleration and viscous terms are negligible and the resulting leading-order behaviour of the Navier-Stokes equations (2) is given by the linearized Euler equations:

$$
\frac{\partial u_{l}}{\partial x}+\frac{\partial v_{l}}{\partial y}=0, \quad \frac{\partial u_{l}}{\partial t}=-\frac{\partial p_{l}}{\partial x}, \quad \text { and } \quad \frac{\partial v_{l}}{\partial t}=-\frac{\partial p_{l}}{\partial y} .
$$

The leading-order behaviour from the kinematic boundary condition gives

$$
v_{l}=\frac{\partial f}{\partial t}, \quad \text { as } \quad y \searrow 0
$$

and therefore following the Green's function approach of Wilson [44] or the complex variable method of Smith et al. [35], the liquid pressure on the droplet free-surface and the free-surface position are related by

$$
\frac{\partial^{2} f}{\partial t^{2}}=\frac{1}{\pi} f_{-\infty}^{\infty} \frac{p_{l, \xi} \mathrm{d} \xi}{x-\xi}
$$

Away from the region of interaction with the gas film (i.e. for $|x| \rightarrow \infty$ and $t \rightarrow-\infty$ ), the droplet free-surface and pressure are given by

$$
f \sim \frac{x^{2}}{2}-t, \quad \text { and } \quad p \rightarrow 0
$$

respectively.

\subsection{Gas film behaviour}

The behaviour of the gas close to the point of touchdown is now investigated. This also occupies a region of horizontal extent $\varepsilon R$. However, the pressure build-up in the gas only starts to interact with the droplet free-surface once the vertical extent of the gas film has closed to $\varepsilon^{2} R$ [35], which motives the definition of dimensionless variables

$$
(x, y, t)=\left(\frac{\tilde{x}}{\varepsilon R}, \frac{\tilde{y}}{\varepsilon^{2} R}, \frac{U \tilde{t}}{\varepsilon^{2} R}\right),
$$

in the gas film. The gas pressure scale must match the liquid pressure scale in order to generate a response in the liquid from the gas pressure build-up, while horizontal gas velocity 
must be an order of $\varepsilon$ larger than the vertical gas velocity given the disparate length scales in (15), in order to conserve gas mass. Therefore

$$
\left(u_{g}, v_{g}, p_{g}, f\right)=\left(\frac{\varepsilon \tilde{u}_{g}}{U}, \frac{\tilde{v}_{g}}{U}, \frac{\varepsilon \tilde{p}_{g}}{\rho_{l} U^{2}}, \frac{\tilde{f}}{\varepsilon^{2} R}\right) .
$$

If $\rho_{g} / \rho_{l} \ll \varepsilon$ and the small parameter $\varepsilon$ is defined to be

$$
\varepsilon \equiv\left(\frac{\mu_{g}}{\rho_{l} U R}\right)^{1 / 3}
$$

in order to balance the pressure gradient with the largest viscous term in the horizontal momentum conservation, then the leading-order behaviour of the Navier-Stokes equations in the gas (4) is given by

$$
\frac{\partial u_{g}}{\partial x}+\frac{\partial u_{g}}{\partial y}=0, \quad 0=-\frac{\partial p_{g}}{\partial x}+\frac{\partial^{2} u_{g}}{\partial y^{2}}, \quad \text { and } \quad 0=-\frac{\partial p_{g}}{\partial y} .
$$

Here (18c), corresponding to conservation of vertical momentum, implies $p_{g}=p_{g}(x, t)$. Several authors have expressed the small parameter $\varepsilon$ in terms of the Stokes number, $\mathrm{St}=$ $\mu_{g} / \rho_{l} U R$ and hence $\varepsilon=\mathrm{St}^{1 / 3}[28,3]$.

The disparate horizontal velocity scales in the liquid droplet and the gas film result in the no-slip and no-penetration boundary conditions

$$
u_{g}=0, \quad \text { and } \quad v_{g}=\frac{\partial f}{\partial t}, \quad \text { on } \quad y=f(x, t),
$$

while the normal stress balance at the droplet-gas interface implies

$$
p_{l}=p_{g}+\sigma \frac{\partial^{2} f}{\partial x^{2}}
$$

where the reciprocal of the reduced Weber number $\sigma=\varepsilon \tilde{\sigma} /\left(\rho_{l} U^{2} R\right)$. For the air-water interface of a droplet of radius $R=1 \mathrm{~mm}$ and impact approach speed $U=0.5 \mathrm{~m} \mathrm{~s}^{-1} ; \sigma=$ 0.01 , indicating surface tension is an unimportant effect for the majority of the free-surface evolution, except perhaps immediately prior to touchdown when the free-surface curvature increases and attempts to form a cusp at the instant of touchdown (refer to the free-surface profiles of Purvis and Smith [31] and $\S 5$ herein as touchdown approaches). Surface tension will be neglected until $\S 5$, to enable cushioning to be investigated in the absence of surface tension. With this simplification $p(x, t) \equiv p_{g}(x, t)=p_{l}(x, 0, t)$ will be used to denote the common pressure across the gas film and on the droplet free-surface $f=f(x, t)$.

\subsection{Gas behaviour in the substrate}

The porous substrate is envisaged as an approximation of a regular array of closely spaced vertical pillars as shown in figure 1 (right). Regular arrays of vertical pillars are commonly used in experiments in order to generate superhydrophobic surfaces [1,24,27,42]. For the experiments of Maitra et al. [26] in which entrapped gas bubbles are observed in the substrate, the height of the pillars forming the substrate $\tilde{h}$ is of the order of $10 \mu \mathrm{m}$.

To couple the gas film behaviour to the flow in the porous substrate, a substrate height $\tilde{h}=$ $\varepsilon^{2} R h$, for a non-dimensional substrate height $h=\mathrm{O}(1)$, is assumed. This restricts interest 
to cases where the substrate height is of equal size to the gas film height at the onset of cushioning. For substrate pillars of $10 \mu \mathrm{m}$ and a water droplet in air, if the radius of the droplet $R=1 \mathrm{~mm}$ and impact speed $U=0.5 \mathrm{~m} \mathrm{~s}^{-1}$, then this gives a non-dimensional substrate height $h=8.96$, which suggests the chosen non-dimensionalization of the substrate height is appropriate for modelling the experiments of Maitra et al. [26]. However, there are many practical examples, such as droplets landing on soil, where the height of the porous substrate is much greater than this. Models appropriate for thicker substrates are briefly discussed in $\S 6$, but a full analysis of these cases is beyond the scope of this paper.

The vertical length scale in the substrate is chosen to match the substrate height, while the horizontal length scale matches that in the gas film and liquid droplet. Hence nondimensional length and time scales

$$
(x, y, t, h)=\left(\frac{\tilde{x}}{\varepsilon R}, \frac{\tilde{y}}{\varepsilon^{2} R}, \frac{U \tilde{t}}{\varepsilon^{2} R}, \frac{\tilde{h}}{\varepsilon^{2} R}\right),
$$

can be defined. Of particular interest is the $\mathrm{O}(\varepsilon R)$ horizontal length scale associated with the porous media and the gas-cushioning interactions, which for a water droplet of radius $1 \mathrm{~mm}$ impacting at $0.5 \mathrm{~m} \mathrm{~s}^{-1}$ is a distance of $33 \mu \mathrm{m}$. The vertical pillars in the substrates used by Maitra et al. [27] are have horizontal separation of $4.5 \mu \mathrm{m}$ and therefore there are 7 pillars per unit non-dimensional horizontal length. This is on the lower limit of the acceptable number of pillars to considered the substrate as a porous medium. However, as there is significant interest in substrates with much more finely spaced pillars, the assumption that the substrate is a porous medium will be used as a first step in modelling this complex phenomena. It is also noted that larger and slower droplets will also improve the porous media approximation.

For air-cushioned impacts between a droplet and an impermeable flat plate a cusp develops in the free surface, which touches down at a single point [35]. If touchdown at a single point is observed in impacts with porous substrates, then on a horizontal length scale local to the touchdown point, the free surface will hit either a pillar or an interstitial void. Hence if touchdown at a point is observed with a porous substrate, then for some region about the touchdown point it is inappropriate to consider the substrate as a porous media. However, for the larger scale dynamics of bubble development and entrainment, treating the substrate as a porous medium can be justified. An alternative approach, which models the larger pillars as individual roughness elements in gas cushioned impacts is described in $\S 4$, extending the approach of Hicks and Purvis [15] who considered individual roughness elements in isolation. Surface roughness has also been considered as an extension to Wagner impact theory for droplet impacts in a passive gas phase [11].

In order to conserve mass in the substrate, velocity scales separated by a factor of $\varepsilon$ are required, while the pressure induced in the substrate must match that in both the gas film and the liquid droplet, and therefore the non-dimensional substrate velocities and pressure can be expressed as

$$
\left(u_{s}, v_{s}, p_{s}\right)=\left(\frac{\varepsilon \tilde{u}_{s}}{U}, \frac{\tilde{v}_{s}}{U}, \frac{\varepsilon \tilde{p}_{s}}{\rho_{l} U^{2}}\right)
$$

With the definition of these non-dimensional variables, the gas motion in the substrate is governed by

$$
\frac{\partial u_{s}}{\partial x}+\frac{\partial v_{s}}{\partial y}=0, \quad u_{s}=-\frac{\rho_{l} U K}{\varepsilon \mu_{g} R} \frac{\partial p_{s}}{\partial x}, \quad \text { and } \quad v_{s}=-\frac{\rho_{l} U K}{\varepsilon^{3} \mu_{g} R} \frac{\partial p_{s}}{\partial y},
$$


while at $y=0$ the normal stress balance gives $p_{s}(x, 0, t)=p_{g}(x, t)$, and the continuity of normal velocity gives $v_{g}(x, 0, t)=v_{s}(x, 0, t)$. At the interface the scaled Beaver-Joseph condition (8) gives

$$
\frac{K^{1 / 2}}{\varepsilon^{2} \alpha R} \frac{\partial u_{g}}{\partial y}=u_{g}-\delta u_{s}
$$

\subsection{Coupled droplet, gas film and substrate flow behaviour}

In order to couple the gas film and liquid droplet to the gas flow in the porous substrate, an effective permeability

$$
k=\frac{\rho_{l} U K}{\varepsilon \mu_{g} R},
$$

is defined. For a water droplet of radius $R=1 \mathrm{~mm}$ in air with impact speed $U=0.5 \mathrm{~m} \mathrm{~s}^{-1}$, an actual substrate permeability $K=10 \mu \mathrm{m}^{2}$, (which is characteristic of a range of porous media), corresponds to an effective permeability $k=8$. With this effective permeability defined, the Darcy flow velocities are

$$
u_{s}=-k \frac{\partial p_{s}}{\partial x}, \quad \text { and } \quad v_{s}=-\frac{k}{\varepsilon^{2}} \frac{\partial p_{s}}{\partial y},
$$

while the pressure satisfies

$$
\frac{\partial^{2} p_{s}}{\partial x^{2}}+\frac{1}{\varepsilon^{2}} \frac{\partial^{2} p_{s}}{\partial y^{2}}=0
$$

If $k \ll 1$ or $\varepsilon^{-2} k \ll 1$, then to leading order the substrate is impermeable to flow in the $x$ or $y$ direction, respectively. For $k \ll 1$, this produces a condition on the impact velocity

$$
U \ll \frac{\mu_{g} R^{1 / 2}}{\rho_{l} K^{3 / 4}},
$$

below which the substrate appears impermeable in the horizontal direction to leading order. The second condition $\varepsilon^{-2} k \ll 1$, leads to a maximum velocity

$$
U \ll \frac{\mu_{g}}{\rho_{l} K^{1 / 2}},
$$

below which there is no vertical gas penetration to leading order, and the impermeable models in [35,15] still apply. For a water droplet of radius $R=1 \mathrm{~mm}$ in air, which impacts a substrate of permeability $K=10 \mu \mathrm{m}^{2}$, the horizontal permeability condition gives the impact velocity condition $U \ll 0.1 \mathrm{~m} \mathrm{~s}^{-1}$, while the vertical permeability condition gives the impact velocity condition $U \ll 0.006 \mathrm{~m} \mathrm{~s}^{-1}$ and therefore for impact speeds equal to or greater than this substrate permeability is expected to play a significant role.

In terms of the effective permeability the Beavers-Joseph condition (24) has the form

$$
\frac{k^{1 / 2}}{\alpha} \frac{\partial u_{g}}{\partial y}=u_{g}-\delta u_{s}
$$

There is very limited experimental evidence supporting the use of the Beavers-Joseph condition on length scales that are as small as those found in the current application. However, the 
Beavers-Joseph coefficient $\alpha$ takes $\mathrm{O}(1)$ values across the limited available experimental parameter regimes. However, further experimental evidence is required to assess its suitability as a boundary condition over the very small length scales considered herein. Saffman [34] showed that the dimensional Beavers-Joseph boundary condition (8) has the form

$$
\tilde{u}_{g}=\frac{K^{1 / 2}}{\alpha} \frac{\partial \tilde{u}_{g}}{\partial \tilde{y}}+\mathrm{O}(K)
$$

where $\tilde{u}_{s}$ in (8) is $\mathrm{O}(K)$ and can be neglected at leading order for $K$ small. This expression suggests a hierarchy of possible boundary conditions in which first flow in substrate, and then additionally velocity shear in the gas as neglected to leave the no-slip boundary condition $\tilde{u}_{g}=0$ as the simplest possible boundary condition. The effect of this hierarchy of boundary conditions on pre-impact gas cushioning will be investigated further in \$A. However, the non-dimensional Beavers-Joseph equation (30) will be adopted here.

Following the approach developed by Knox et al. [21] for the coupled squeeze film and porous substrate flow driven by an approaching flat plate, the pressure in the substrate is expanded as

$$
p_{s}(x, y, t)=p(x, t)+\varepsilon^{2} P(x, y, t) \text {, }
$$

where the leading-order gas pressure in the substrate matches that in the gas film because of the normal stress balance at the surface of the substrate. From (27) and the no-penetration condition at $y=-h$, the correction to substrate pressure $P(x, y, t)$ satisfies

$$
\frac{\partial P}{\partial y}=-(y+h) \frac{\partial^{2} p}{\partial x^{2}} .
$$

In particular the Darcy velocities on the substrate interface are

$$
u_{s}(x, 0, t)=-k \frac{\partial p}{\partial x}, \quad \text { and } \quad v_{s}(x, 0, t)=k h \frac{\partial^{2} p}{\partial x^{2}}
$$

Integrating in the horizontal momentum equation in the gas film (18b) and applying the Beavers-Joseph condition (30) gives a horizontal gas velocity

$$
u_{g}=-\frac{(f-y)\left[\left(\alpha f+k^{1 / 2}\right) y+k^{1 / 2} f+2 k \alpha \delta\right]}{2\left(\alpha f+k^{1 / 2}\right)} \frac{\partial p}{\partial x},
$$

after substituting for the horizontal Darcy velocity at the surface of the substrate. Now integrating the gas mass conservation equation across the gas film gives

$$
\frac{\partial f}{\partial t}=\frac{1}{12} \frac{\partial}{\partial x}\left[\frac{f^{2}\left(\alpha f^{2}+4 k^{1 / 2} f+6 k \alpha \delta\right)}{\alpha f+k^{1 / 2}} \frac{\partial p}{\partial x}\right]+k h \frac{\partial^{2} p}{\partial x^{2}},
$$

when the vertical Darcy velocity is included. Using the normal stress balance (20), a system of equations for $f(x, t)$ and $p(x, t)$ is completed by the boundary integral (13), which reads

$$
\frac{\partial^{2} f}{\partial t^{2}}=\frac{1}{\pi} f_{-\infty}^{\infty} \frac{p_{\xi} \mathrm{d} \xi}{x-\xi}
$$

Equations (36) and (37) are solved numerically. The integro-differential equation (37) is evaluated via a complex Fourier series ( $\operatorname{see} \S \mathrm{B}$ for details with the parameter $\sigma=0$ ). This 


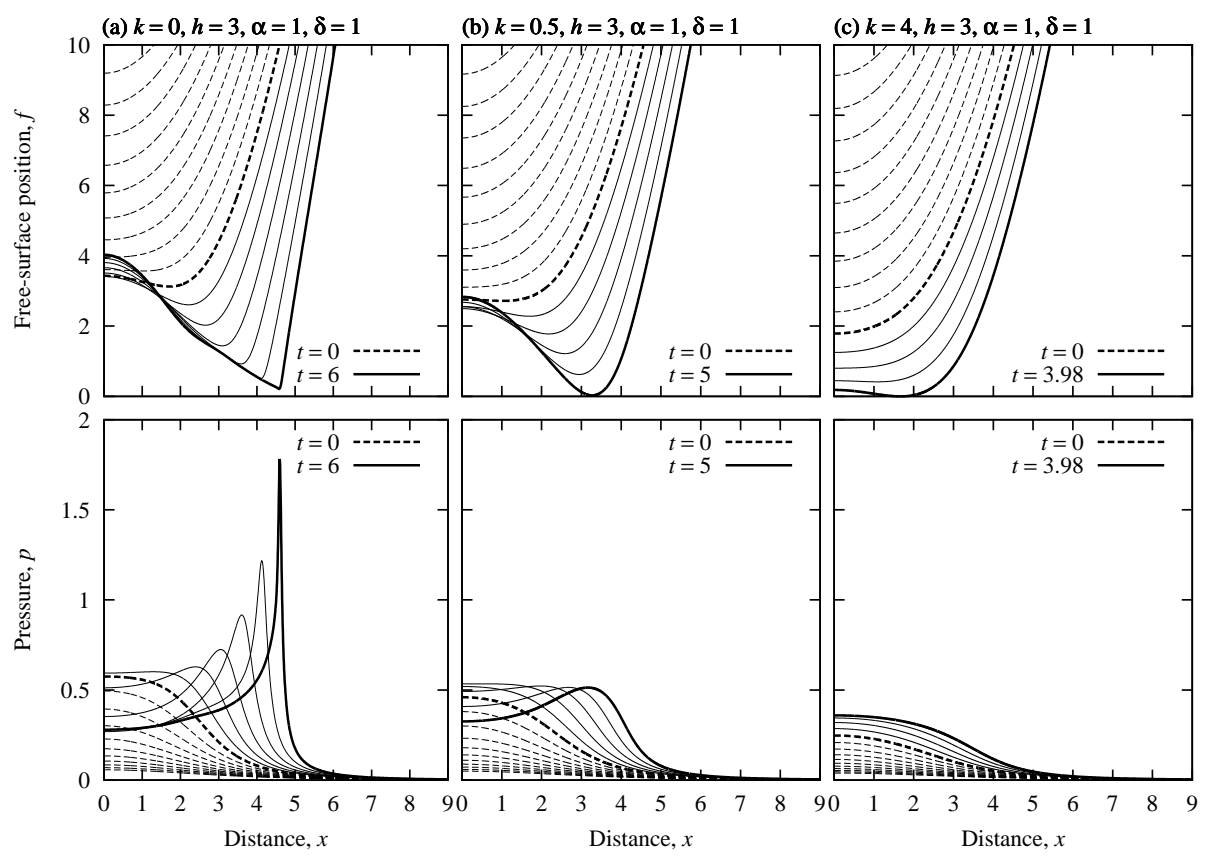

Fig. 2: The evolution of the droplet free-surface (top) and the gas film pressure (bottom) for gas cushioned impacts for (a) $k=0$ (an impermeable flat plate), (b) $k=8$ and (c) $k=$ 16. In all cases $h=5, \alpha=1$ and $\delta=1$. Profiles are shown at non-dimensional integer time increments, except for the final profile which is shown at touchdown. Dotted lines correspond to times at or before $t=0$ (the touchdown time in a vacuum), while solid lines correspond to positive delayed touchdown time increments.

yields a simplified equation relating the free-surface shape $f$ and the pressure $p$. We discretise (36) using implicit finite differences, which enables the pressure to be calculated from a known free-surface shape. An iteration is then performed between these two equations; convergence of the solution to within $10^{-6}$ typically takes a limited number of iterations. This method offers a considerable saving in simulation time over previous work $[15,16]$ that discretised (37), particularly when surface tension is included.

\section{Cushioned impact with a porous substrate of shallow height}

Figure 2 shows the free-surface evolution (top) and the corresponding pressures (bottom) for $h=3, \alpha=1, \delta=1$ and a range of values of effective permeability $k$. In 2(a) the case corresponding to $k=0$, an impermeable flat plate is shown. The behaviour here matches exactly that observed above a smooth flat impermeable plate, which is to be expected as the squeeze film equation

$$
\frac{\partial f}{\partial t}=\frac{1}{12} \frac{\partial}{\partial x}\left[f^{3} \frac{\partial p_{g}}{\partial x}\right]
$$




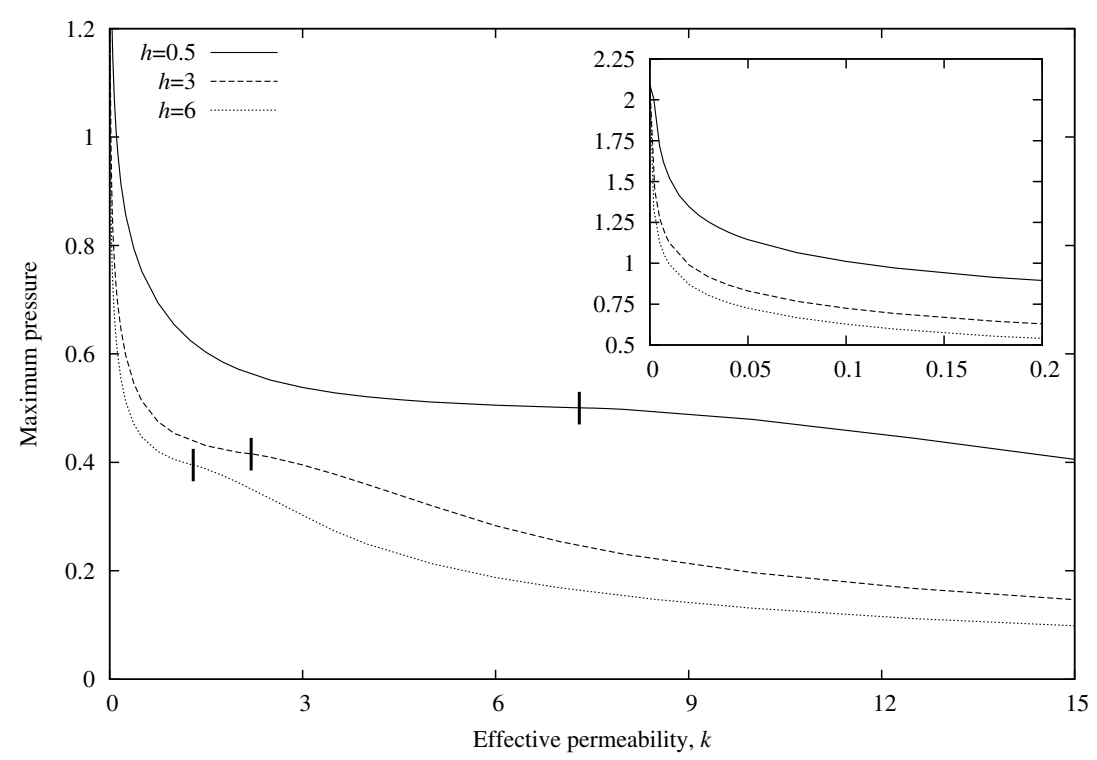

Fig. 3: The gas film pressure at touchdown against effective permeability $k$ for three depths $h=0.5,3,6$. The inset shows behaviour for small $k$. The vertical line fragments denote the transition from pressure profiles having two maxima (left) to having a single maximum at $x=0$ (right). The other parameters are fixed as $\alpha=1$ and $\delta=1$.

of the model of Smith et al. [35] is recovered from equation (36) in the limit $k \rightarrow 0$. The model of Smith et al. also incorporates the integral equation (37). In this case there is an initial pressure build-up underneath the descending droplet causing the descending droplet free-surface to decelerate. Eventually the decelerated droplet free-surface directly below the centre of the droplet is overtaken by the surrounding free surface, with the pressure profiles similarly bifurcating to produce maxima where the separation between free surface and substrate are least. Touchdown then goes on to occur around a ring some horizontal distance away from the point directly below the centre of an air bubble, leading to a trapped pocket of gas, which subsequently may evolve to form a bubble, as observed in experiments [4, $6,38]$. In a vacuum, the initial touchdown would occur at time $t=0$. However, with gas cushioning the profiles at $t=0$ (denoted by a thick dashed line), have yet reach touchdown due to the deceleration of the droplet free surface and therefore with gas cushioning present the touchdown is delayed.

The evolution of the droplet free-surface and the pressure for increasing values of $k$ are shown in figure 2(b) and (c). In figure 2(b), the effective permeability $k=0.5$, which provides additional pathways through which the gas can escape from beneath the droplet. This results in a lower gas pressure build-up, a less decelerated droplet free-surface and ultimately a smaller pocket of gas is trapped. This gas pocket is still connected to the far field via pathways through the porous substrate. For a further increase to $k=4$ in figure 2(c), a small bubble is still trapped, but at the instant of touchdown the pressure profiles have yet to bifurcate and one global maximum remains. Further increases in $k$ produce cases where there is no trapped bubble, with touchdown occurring at a single point below the centre 


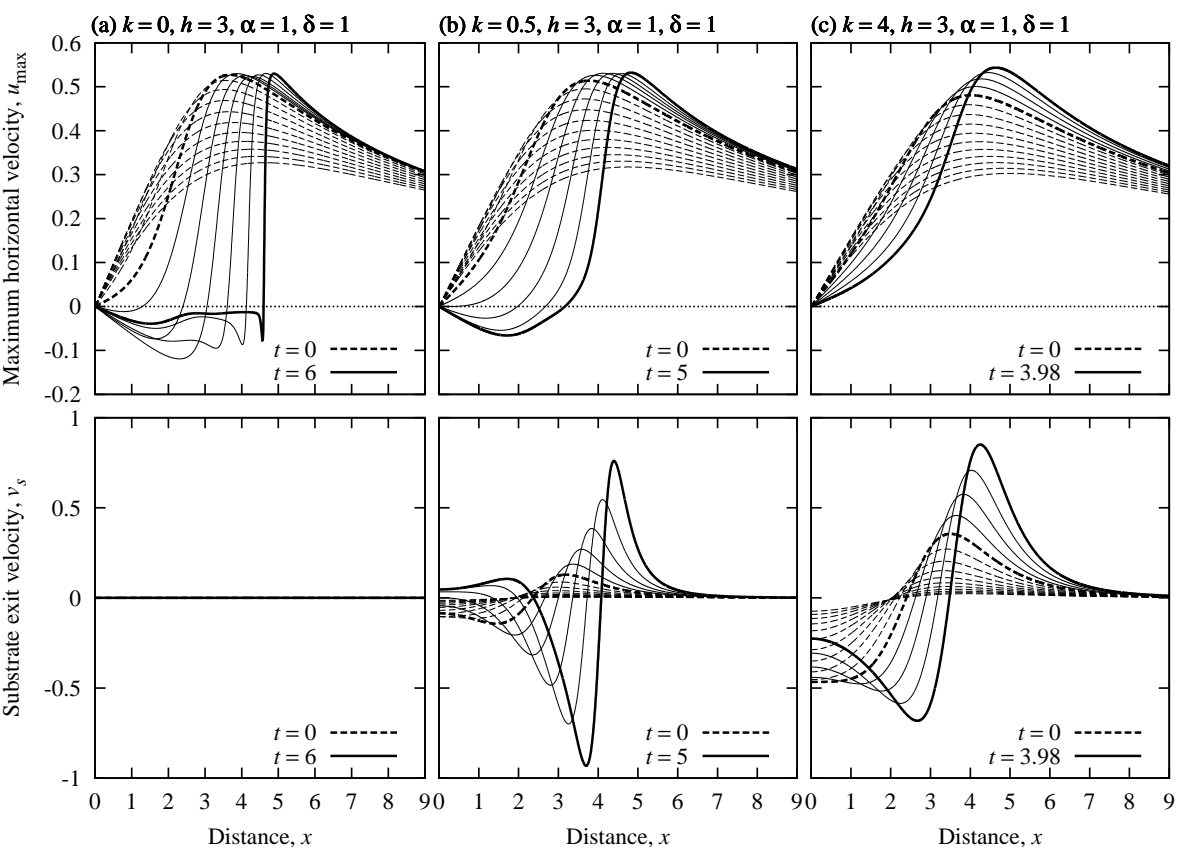

Fig. 4: As figure 2, except showing the evolution of the maximum horizontal gas film velocity (top) and the substrate exit velocity (bottom).

of the droplet. The maximum calculated pressure, found as touchdown is reached, against permeability is shown in figure 3 for various porous layer depths. The maximum pressure can be seen to decrease with increasing permeability. The minimum rate of the pressure decrease with permeability coincides with the onset of bifurcated pressure profiles, with higher rates of pressure change observed for both large and smaller $k$.

The free-surface profiles for $k>0$ actually reach touchdown, while for the impermeable plate case the numerical solution of the parabolic equation (38) fails to converge just prior to touchdown. Numerical convergence is harder to achieve for the flat plate case as the pressures and pressure gradients just before touchdown are larger than their counterparts with a permeable substrate. Related to this, the gas film equation for a flat plate (38) is parabolically degenerate, as the diffusion coefficient tends to zero for $f$ small. For a permeable substrate, this is regularized by any non-zero product $k h$ in (36), with the behaviour for $f$ small being given by the parabolic equation

$$
\frac{\partial f}{\partial t}=k h \frac{\partial^{2} p_{g}}{\partial x^{2}}
$$

This makes it easier for the droplet free-surface to touchdown on a porous substrate. However, in order to apply (36) right up to the instant of touchdown, the length scale over which the substrate can be legitimately assumed to be a porous medium needs to be much smaller than the contact region. 
The maximum horizontal gas velocity is obtained in the gas film at a height

$$
y_{\max }=\frac{f}{2}-\frac{k^{1 / 2} f+2 \alpha k \delta}{2\left(\alpha f+k^{1 / 2}\right)},
$$

which is slightly below the channel centre line as a result of the horizontal slip velocity at the substrate surface. The maximum horizontal gas velocity

$$
u_{g, \max }=\frac{\left[\left(\alpha f+k^{1 / 2}\right) f+k^{1 / 2} f+2 \alpha k \delta\right]^{2}}{8\left(\alpha f+k^{1 / 2}\right)^{2}} \frac{\partial p}{\partial x},
$$

is shown if figure 4 (top) for the same cases shown in figure 2. For $t<0$ the horizontal gas velocities are positive for all $x>0$ as gas is forced out horizontally, away from the path of the approaching droplet. In figure 2(c) a small bubble is trapped, but there is no bifurcation of the pressure, and consequently in figure 4(c) gas is still driven out horizontally from beneath the droplet right up to the instant of touchdown as a result of the monotonically decreasing pressures for $x>0$. As the effective permeability is decreased in 4(a) and (b), the pressure profiles bifurcate and have two maxima. For positive $x$ values less than the location of the pressure maxima, the horizontal gas velocities are negative and (driven by the pressure gradient), gas is flows towards the centre of the bubble. For $x$ values greater the location of the pressure maxima, the gas is still forced horizontally outwards away from the oncoming droplet.

The vertical gas velocity as it exits the substrate is given by (34b), and takes positive (negative) values when gas is exiting (entering) the substrate from the gas film. The substrate exit velocity is shown in figure 4 (bottom) for the same parameter cases as are shown in figure 2. As expected for the impermeable flat plate $k=0$ shown in 4(a), there is no gas penetration into the substrate. As the effective permeability increases in figures 4(b) and (c), there is a flow of gas into and out of the substrate. Initially (for $t<0$ ), gas enters the substrate below the centre of the droplet, driven by the approaching droplet. At horizontal distances larger than that corresponding to the inflection point in $p$, gas passes from the substrate back into the gas film, in order to conserve the total mass of gas in the substrate. For $t \gtrsim 2$ in figure 4(b) gas exits the substrate immediately below the centre of the droplet. The acts to increase the volume of the gas pocket trapped in this region.

From the gas film non-dimensionalization (15) and (16), in two spatial dimensions the dimensional horizontal extent $\tilde{x}_{p}$ and characteristic vertical height $\tilde{f}_{p}$ of the gas pocket are given by

$$
\tilde{x}_{p}=\varepsilon R x_{p}=\frac{\mu_{g}^{1 / 3} R^{2 / 3}}{\rho_{l}^{1 / 3} U^{1 / 3}} x_{p}, \quad \text { and } \quad \tilde{f}_{p}=\varepsilon^{2} R f_{p}=\frac{\mu_{g}^{2 / 3} R^{1 / 3}}{\rho_{l}^{2 / 3} U^{2 / 3}} f_{p},
$$

respectively. Here the non-dimensional prefactors $x_{p}$ and $f_{p}$ can be determined from the free-surface profiles at touchdown. From these formulae it is evident that both the horizontal extent and vertical height of the trapped gas pocket increases with increases in $R$, as the droplet has a greater free surface to interact with the gas cushion, and decreases as the impact speed $U$ increases, as there is less time for the free surface to respond prior to impact. In impacts with rigid impermeable solids the prefactors $x_{p}$ and $f_{p}$ are constant. However, with a porous substrate these quantities become functions of the substrate parameters $k, h, \alpha$ and $\delta$. The dependence of the prefactor $x_{p}$ on the effective permeability $k$ and effective porous substrate height $h$ is shown in figure 5(a). Increases in either $k$ or $h$ reduce the horizontal 
(a) Horizontal bubble extent, $x$

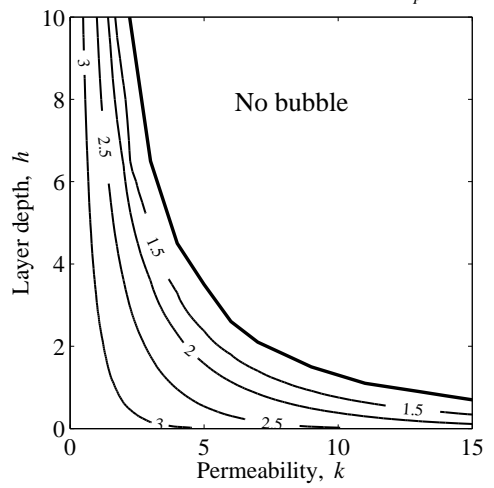

(c) Touchdown time, $t$

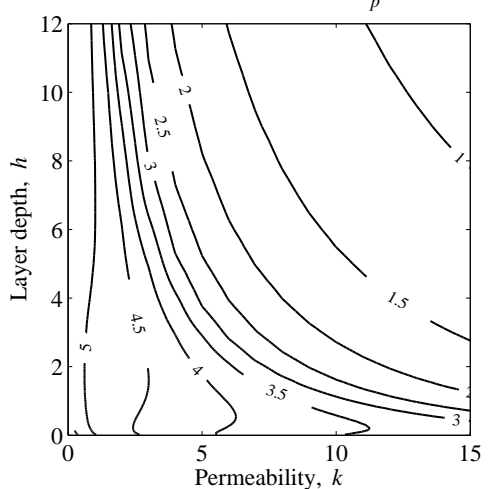

(b) Bubble area, $A_{p}$

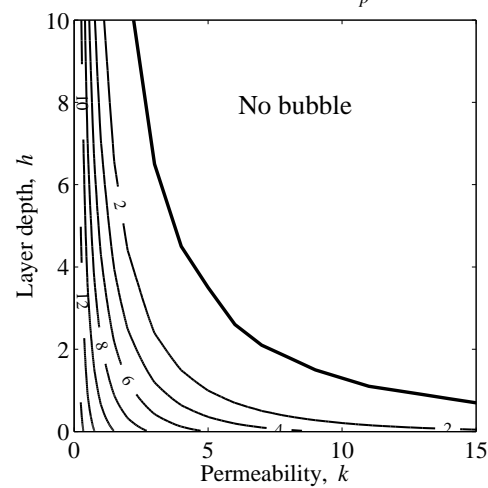

(d) Load, $L_{p}$

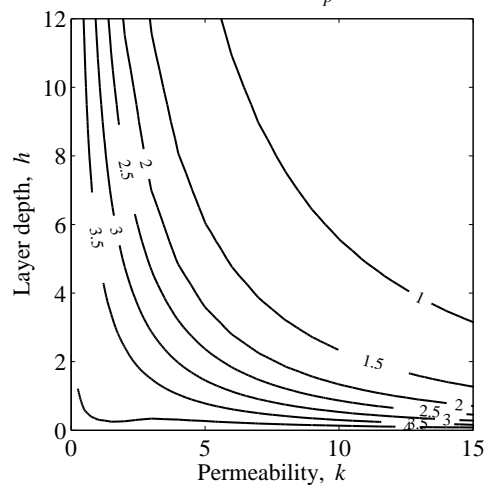

Fig. 5: Variations in (a) horizontal bubble extent $x_{p}$, (b) bubble area $A_{p}$, (c) touchdown time $t_{p}$ and (d) load at touchdown $L_{p}$, as the effective permeability $k$ and substrate height $h$ varies.

extent of the trapped bubble, with the largest bubbles being trapped by an impermeable flat plate. The thick black line shown in figure 5(a) is the curve $x_{p}=0$, which physically corresponds to the transition between cases which do and do not trap a gas pocket. Above this line the droplet free-surface will not decelerated sufficiently prior to touchdown to cause the bifurcations and multiple free-surface minima. In this case touchdown will occur at a single point immediately below the centre of the droplet.

In axisymmetry equation (42a) would give the dependence of the initial radius of the gas pocket on $R$ and $U$, although numerical calculations show that the appropriate nondimensional prefactor differs considerably from that calculated in two dimensions. The dependence of the initial gas pocket radius upon $R, U$ and the non-dimensional prefactor has been corroborated experimentally in axisymmetric impacts of an impermeable solid body 
with an initially undisturbed liquid free-surface [14]. The two-dimensional bubble area $\tilde{A}_{p}$, and axisymmetric bubble volume $\tilde{V}_{p}$, can thus be obtained as the product of the horizontal and vertical length scales:

$$
\tilde{A}_{p}=\varepsilon^{3} R^{2} A_{p}=\frac{\mu_{g} R}{\rho_{l} U} A_{p}, \quad \text { and } \quad \tilde{V}_{p}=\varepsilon^{4} R^{3} V_{p}=\frac{\mu_{g}^{4 / 3} R^{5 / 3}}{\rho_{l}^{4 / 3} U^{4 / 3}} V_{p}
$$

The dependence of $A_{p}$ upon $k$ and $h$ is illustrated in figure 5(b) and mirrors the dependence seen with the behaviour of $x_{p}$. Again the thick line marks the transition to cases not forming a trapped gas pocket.

From (15), the time delay until touchdown is given by

$$
\tilde{t}_{p}=\frac{\varepsilon^{2} R}{U} t_{p}=\frac{\mu_{g}^{2 / 3} R^{1 / 3}}{\rho_{l}^{2 / 3} U^{5 / 3}} t_{p}
$$

again indicating the length of the delay is increased with droplet radius $R$ and decreased with impact speed $U$. For a porous substrate the non-dimensional prefactor $t_{p}$ depends upon $k$ and $h$ and this dependence is shown in figure 5(c). For the majority of the parameter regime shown the time delay before touchdown is decreased by increasing either $k$ or $h$, as the obstructions preventing gas penetrating the substrate fall as $k$ and $h$ increase. However, there is a small region for $h \approx 0.75$ in which increases in substrate height actually lead to increased delays in the touchdown time. This may be of interest when optimizing the superhydrophobic properties of substrate. However further investigation with numerical calculations in axisymmetry and experiments are required to investigate this region further.

Figure 2 showed that the gas pressures are reduced as the substrate effective permeability increases giving additional pathways through which the gas can escape. The nondimensional impact load

$$
L_{p}=\int_{-\infty}^{\infty} p\left(x, t_{p}\right) \mathrm{d} x
$$

which is related to the dimensional impact load $\tilde{L}_{p}=\rho_{l} U^{2} R L_{p}$, is shown in figure 5(d) for a range of different values of $k$ and $h$. This figure shows that the pre-impact load factor $L_{p}$ is decreased by increases in either $k$ or $h$, as a consequence of lower induced gas pressures. Consequently if droplets of equal size and impact speed hit an impermeable and a permeable substrate, then the permeable substrate experiences a lower load, a property which may be of use when producing long lasting coatings for surfaces.

To summarize the results of this section the pre-impact cushioning behaviour of a twodimensional droplet of water of radius $R=1 \mathrm{~mm}$ and impact approach speed $U=0.5 \mathrm{~m} \mathrm{~s}^{-1}$ in air is considered. For a flat rigid impermeable plate the prefactors $x_{p}=4.55$ and $A_{p}=$ 18.76, indicate that the initial horizontal extent and area of the trapped bubble are $\tilde{x}_{p}=$ $152 \mu \mathrm{m}$ and $\tilde{A}_{p}=700 \mu \mathrm{m}^{2}$, respectively. If the same droplet impacts a shallow porous layer of permeability $K=2.5 \mu \mathrm{m}^{2}$ and height $\tilde{h}=5 \mu \mathrm{m}$, then the corresponding initial horizontal extent and area of the trapped bubble are $\tilde{x}_{p}=79 \mu \mathrm{m}$ and $\tilde{A}_{p}=71 \mu \mathrm{m}^{2}$, respectively. This is a $48 \%$ reduction in the horizontal extent of the initial trapped bubble radius, while further increases in substrate permeability or height can eliminate the formation of a trapped bubble entirely. 

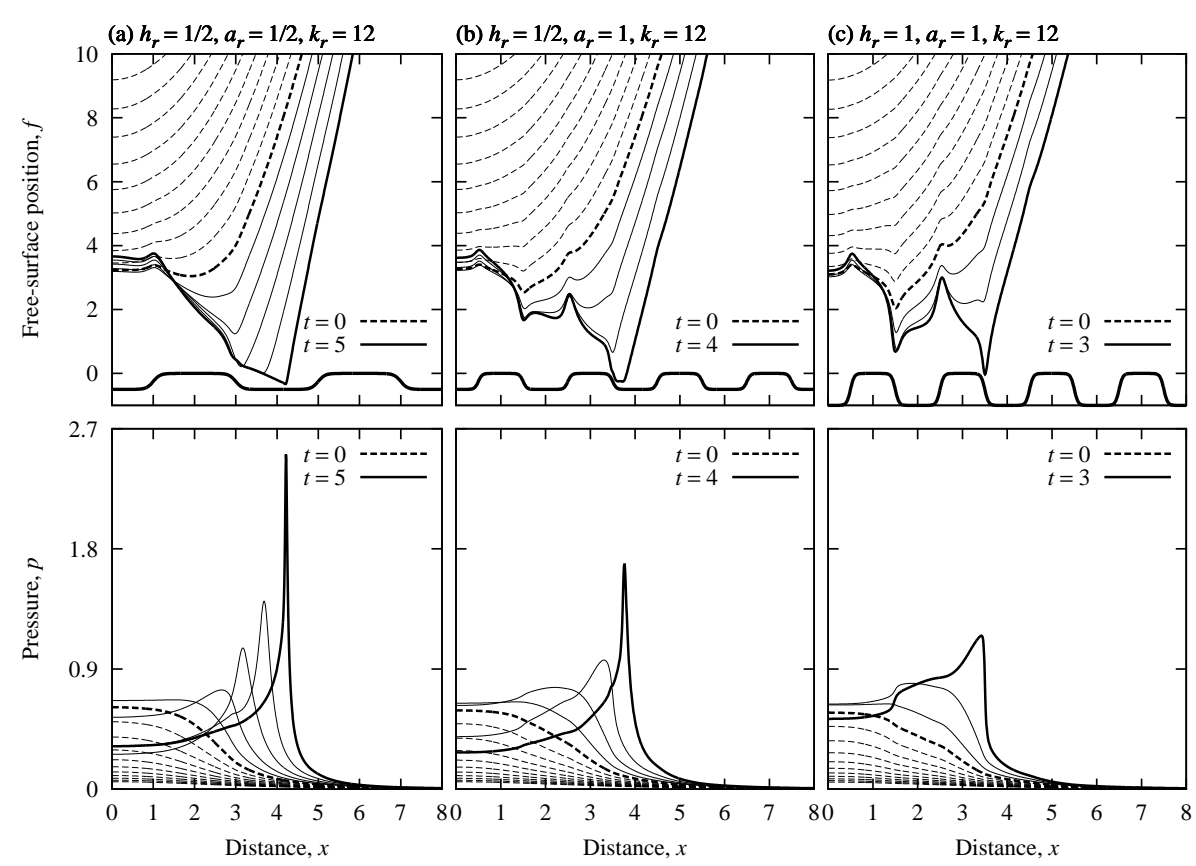

Fig. 6: The evolution of the droplet free-surface (top) and the gas film pressure (bottom) for gas cushioned impacts with a textured surface with (a) $h_{r}=1 / 2, a_{r}=1 / 2$ and $k_{r}=$ 12, (b) $h_{r}=1 / 2, a_{r}=1$ and $k_{r}=12$, and (c) $h_{r}=1, a_{r}=1$ and $k_{r}=12$. Profiles are shown at non-dimensional integer time increments. Dotted lines correspond to times at or before $t=0$ (the touchdown time in a vacuum), while solid lines correspond to positive delayed touchdown time increments.

\section{Cushioned impact with a textured surface}

To assess how an array of larger pillars may effect the pre-impact cushioning behaviour, the assumption that the substrate is a shallow porous medium is temporarily abandoned and a lower surface of the gas cushion $s(x)$, which incorporates multiple roughness elements is used. This extends the previous analysis of Hicks and Purvis [15], who investigated individual roughness elements in three-dimensional gas-cushioned droplet impacts. When no-slip and no-penetration conditions $u_{g}=v_{g}=0$ are applied on the surface $s(x)$ of a rough substrate, the lubrication (36) is modified to become

$$
\frac{\partial f}{\partial t}=\frac{1}{12} \frac{\partial}{\partial x}\left[(f-s(x))^{3} \frac{\partial p}{\partial x}\right] .
$$

A substrate roughness

$$
s(x)=\sum_{n=-N}^{N} \frac{h_{r}}{2}\left[\tanh \left(k_{r}\left(a_{r} x-2 n-\frac{1}{2}\right)\right)-\tanh \left(k_{r}\left(a_{r} x-2 n+\frac{1}{2}\right)\right)\right],
$$

is used, which gives a periodic array of indentations to the surface $y=0$ constructed from hyperbolic tangent functions. Here $h_{r}$ is the height of the surface indentations while the 
horizontal extent and slope can be modified by changing $a_{r}$ and $k_{r}$ respectively. Within our long wave formulation of the gas cushioning, combinations of step functions cannot be used and hence true pillars cannot be represented.

Numerical solutions of (46-47) and the usual integral equation for the droplet freesurface (37) are shown in figure 6 for (a) $h_{r}=1 / 2, a_{r}=1 / 2$ and $k_{r}=12$, (b) $h_{r}=1 / 2$, $a_{r}=1$ and $k_{r}=12$, and (c) $h_{r}=1, a_{r}=1$ and $k_{r}=12$. Direct quantitative comparisons with the earlier results for a porous substrate are difficult because the porous substrate formulation relies on the substrate permeability, while only the void fraction is readily available with this formulation involving surface roughness. In this two dimensional formulation of substrate roughness, no mechanism exists for gas to pass from one indentation to the next without passing through $y=0$ and then over the top of a roughness element. This is not the case in the porous substrate or the three-dimensional array of pillars shown in figure 1 (right), where gas can move laterally between adjacent pillars.

When compared to figure 2(a), the free-surface profiles and to a lesser extent the pressure profiles with surface roughness show significant variation at horizontal distances at which surface height varies. The variations in droplet profiles are evident even for times $t>-3$, indicating that individual roughness elements start to effect the droplet profile significantly before touchdown occurs. This indicates that roughness elements are required to have a characteristic horizontal length scale that is much smaller than that of the roughness elements shown here, in order to legitimately consider the substrate as a porous medium.

\section{Cushioned impact with a porous substrate of shallow height and surface tension}

With surface tension included the droplet free-surface evolution integral equation (13) is given by [31]

$$
\frac{\partial^{2} f}{\partial t^{2}}=\frac{1}{\pi} f_{-\infty}^{\infty} \frac{p_{g, \xi}+\sigma f_{\xi \xi \xi} \mathrm{d} \xi}{x-\xi}
$$

This modified integral equation is stiff and direct calculation of the free-surface acceleration requires an impractically small time step. To overcome this, the pressure and the deviation of the liquid free-surface from the undeformed free-surface position $x^{2} / 2-t$, is obtained via a periodic complex Fourier series expansion. Further details of the numerical procedure are given in $\S \mathrm{B}$.

Figure 7 shows a range of gas-cushioned droplet impacts with a permeable substrates with $k=0.5, h=3, \alpha=1$ and $\delta=1$ in which surface tension is included, while figure 8 shows the equivalent droplet impacts with an impermeable flat plate. In both figures, profiles are shown for (a) $\sigma=0.01$, (b) $\sigma=0.1$, and (c) $\sigma=1$. For a water droplet of radius $R=1 \mathrm{~mm}$ and velocity $U=0.5 \mathrm{~m} \mathrm{~s}^{-1}$ impacting a porous substrate cushioned by air, $\sigma=0.01$ and for this case, the profiles in figure 7(a) match very closely those shown in figure 2(b), indicating the effect of surface tension is insignificant in this regime. The differences between the cases $\sigma=0$ and $\sigma=0.01$ is reduced when the droplets hit a permeable substrate than when the droplets hit a flat impermeable plate (see figures 8(a) and 2(a)), although good agreement is still obtained with a flat plate. This is because the droplet freesurface does not form a cusp in an impact with a porous substrate and hence the curvature term in (48) is less significant. This is also evident in the corresponding profiles of the freesurface curvature.

As the value of $\sigma$ increases, droplets hitting a porous substrate continue to reach touchdown. This is in marked contrast to earlier predictions $[31,28]$ and the droplet profiles shown 


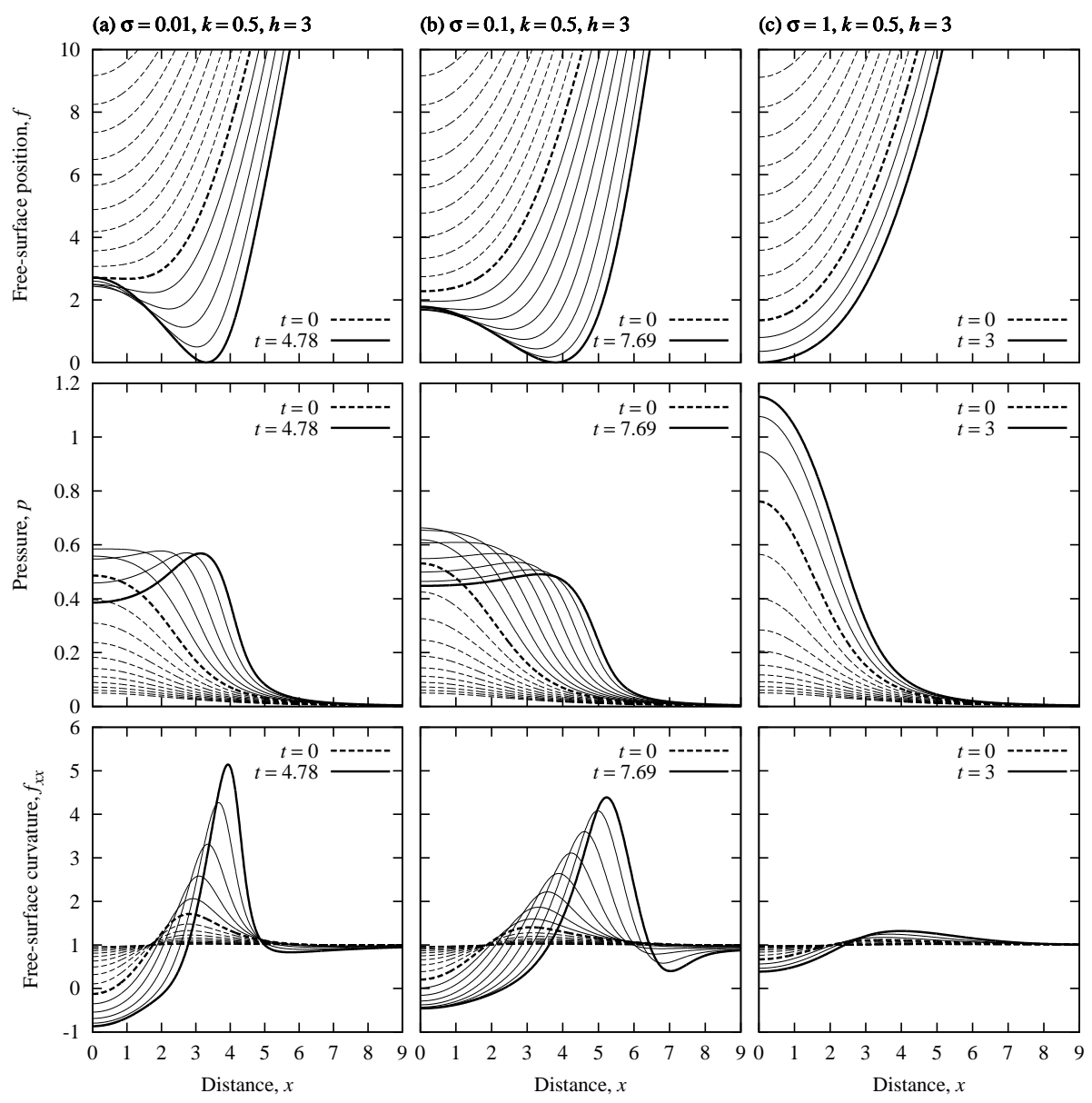

Fig. 7: The evolution of the droplet free-surface (top), pressure (middle) and free-surface curvature (bottom) for (a) $\sigma=0.01$, (b) $\sigma=0.1$ and (c) $\sigma=1$. Profiles are shown at integer time increments except for the final thick solid line which is the profile upon touchdown. In all cases the substrate is a shallow porous layer with $k=0.5, h=3, \alpha=1$ and $\delta=1$.

in figure 8, for impacts with a flat impermeable plate where touchdown isn't predicted in finite time. With a porous substrate and moderate surface tension, there is less evidence of capillary waves on the droplet free-surface at touchdown, which can be clearly observed in impacts with a flat plate (see the free-surface curvature).

For $\sigma=1$ the surface tension is so strong that the droplet fails to deform significantly prior to touchdown with the porous substrate, as indicated by both the lack of trapped gas bubble and the small range of the free-surface curvature values. In this case touchdown occurs at an earlier time instant than both in impacts with a porous substrate and lower surface tension levels, or in impacts with a flat plate at an equivalent surface tension level. This leads to the counter intuitive conclusion that a textured substrate, which is perhaps 

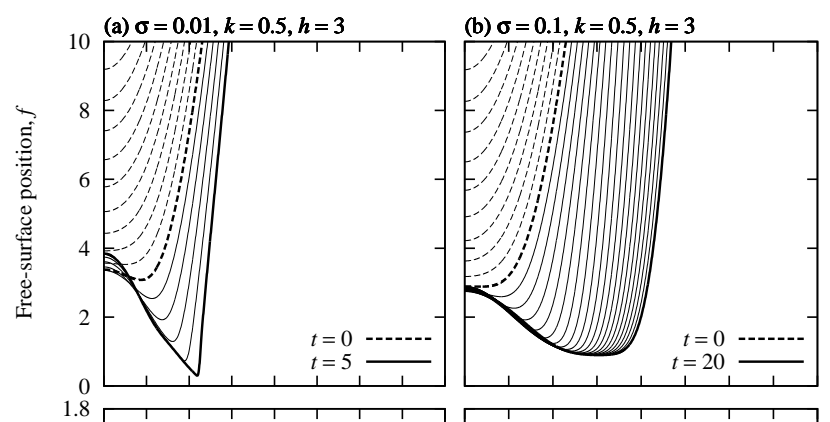

(c) $\sigma=1, k=0.5, h=3$
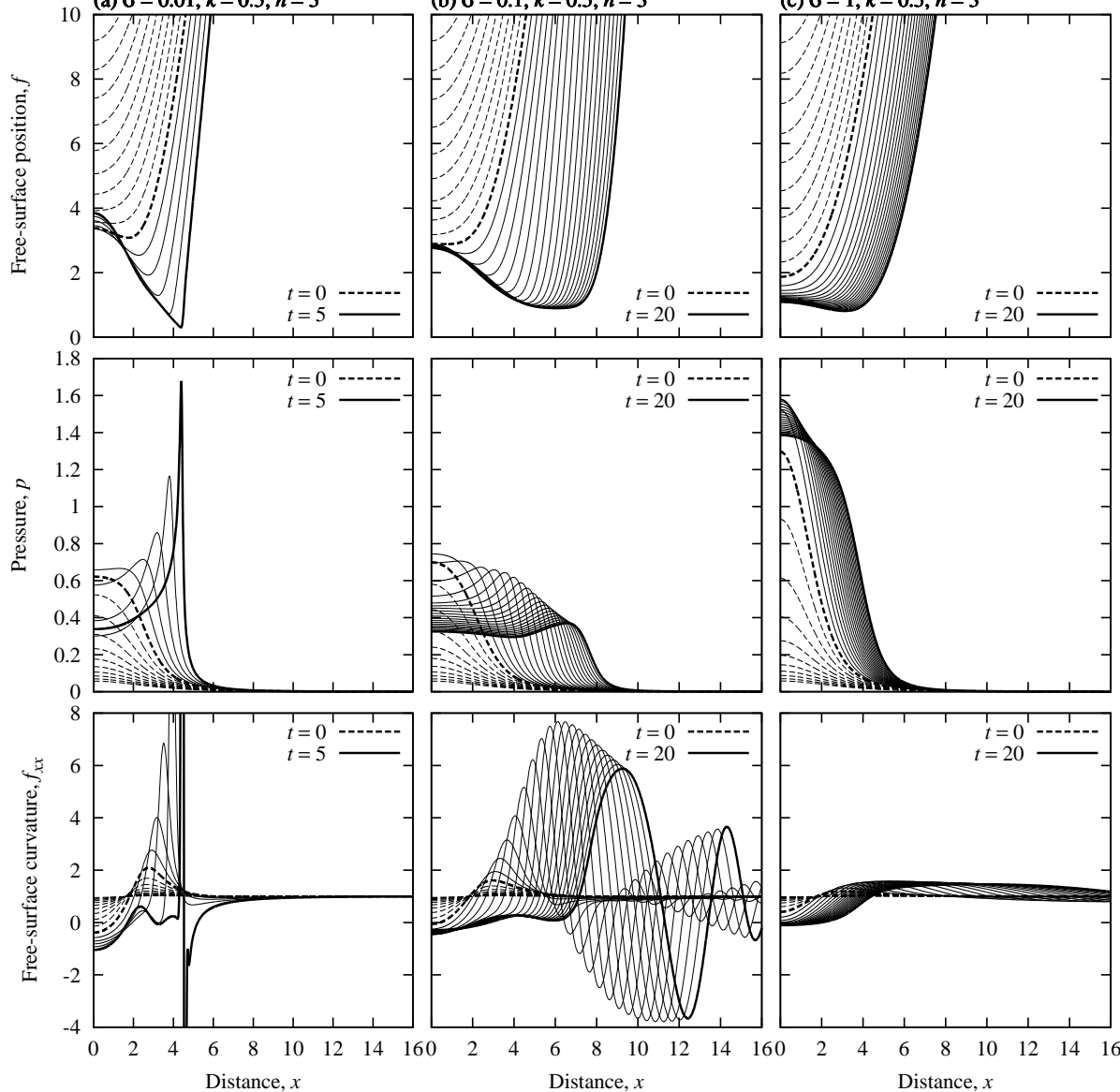

Fig. 8: As figure 7, except with a flat impermeable plate.

constructed to have super-hydrophobic properties, and a surfactant laden droplet free-surface can actually act together to promote initial droplet touchdown.

The non-monotonic dependency of the time to touchdown upon $\sigma$ shown in figure 7 is due to a transition from a regime in which the droplet inertia dominates deformation to a regime in which surface tension dominates. To complete the analysis of section 3 , the dimensions of a trapped gas pockets on the impact parameters in the surface tension dominated regime can be predicted, with the analysis for a porous substrate following the analysis of Bouwhuis et al. [3] for a flat impermeable plate. For surface tension dominated droplet deformation, the largest viscous term in the horizontal gas momentum equation (18b) is balanced by the horizontal derivative of the capillary pressure, as a result of the normal stress balance (6b), rather than the horizontal derivative of the liquid pressure. Using the gas 
non-dimensionalization (15-16) this implies

$$
\frac{\mu_{g} U}{\varepsilon_{c}^{5} R^{2}} \sim \frac{\tilde{\sigma}}{\varepsilon_{c} R^{2}},
$$

where the revised small parameter

$$
\varepsilon_{c} \equiv\left(\frac{\mu_{g} U}{\tilde{\sigma}}\right)^{1 / 4},
$$

is obtained by balancing these terms. The small parameter $\varepsilon_{c}$ can also be expressed in terms of the capillary number, defined with the gas viscosity, $\mathrm{Ca}=\mu_{g} U / \tilde{\sigma}$ and hence $\varepsilon_{c}=$ $\mathrm{Ca}^{1 / 4}[3]$.

From the gas non-dimensionalization (15-16) scaling laws for the horizontal extent and vertical height of the gas pocket

$$
\tilde{x}_{p, c}=\varepsilon_{c} R x_{p, c}=\frac{\mu_{g}^{1 / 4} U^{1 / 4} R}{\tilde{\sigma}^{1 / 4}} x_{p, c}, \quad \text { and } \quad \tilde{f}_{p, c}=\varepsilon_{c}^{2} R f_{p, c}=\frac{\mu_{g}^{1 / 2} U^{1 / 2} R}{\tilde{\sigma}^{1 / 2}} f_{p, c}
$$

are obtained for the surface tension dominated regime. As before, for impacts with permeable substrates, the prefactors $x_{p, c}$ and $f_{p, c}$ are functions of the substrate properties, although these are expected to differ from those obtained in section 3 . The corresponding results for the bubble area in two-dimensional flows and the bubble volume in three-dimensions are thus

$$
\tilde{A}_{p, c}=\varepsilon_{c}^{3} R^{2} A_{p, c}=\frac{\mu_{g}^{3 / 4} U^{3 / 4} R^{2}}{\tilde{\sigma}^{3 / 4}} A_{p, c}, \quad \text { and } \quad \tilde{V}_{p, c}=\varepsilon_{c}^{4} R^{3} V_{p, c}=\frac{\mu_{g} U R^{3}}{\tilde{\sigma}} V_{p, c},
$$

while the delay in touchdown is

$$
\tilde{t}_{p, c}=\frac{\varepsilon_{c}^{2} R}{U} t_{p, c}=\frac{\mu_{g}^{1 / 2} R}{\tilde{\sigma}^{1 / 2} U^{1 / 2}} t_{p, c} .
$$

Again the prefactors $A_{p, c}, V_{p, c}$ and $t_{p, c}$ are functions of the substrate parameters. The predictions for the horizontal extent of the bubble (51a) and the bubble volume (52b) have been experimentally verified for droplet impacts with a flat impermeable plate [3]. For surface tension dominated droplet deformations it is notable that the predicted bubble dimensions and volume increase with impact velocity $U$, in contrast to the earlier predictions in the inertia dominated droplet deformation regime where the gas bubble volume decreased with $U$.

The surface tension dominated regime is associated with smaller and slower droplets than discussed previously, where the ability of surface tension to retain a spherical droplet is greater. As the speed increases the transition from capillary dominated deformation to droplet inertia dominated droplet deformation occurs when the gas pressure, the liquid pressure and the capillary pressure have equal magnitude in the normal stress balance (6b). This occurs when $\varepsilon \sim \varepsilon_{c}$ and hence while a porous substrate will decrease all bubble volumes compared to a flat impermeable plate, the impact velocity associated with the maximum gas bubble volume of Bouwhuis et al.

$$
U \sim \frac{\mu_{g}^{1 / 7} \tilde{\sigma}^{3 / 7}}{\rho_{l}^{4 / 7} R^{4 / 7}},
$$

is unchanged by the presence of a thin porous substrate. 


\section{Discussion and future work}

Novel models describing the pre-impact gas cushioning of droplets approaching impact with a thin layer of porous media bounded below by an impermeable base have been created and analyzed via the numerical solution of a system of coupled integro-differential equations. The thin layer of porous media is an idealized description of a dense array of regular pillars as often found in in droplet impacts with superhydrophobic and superhydrophilic textured surfaces. The model developed is equally applicable in any other situation where a droplet impacts a layer of porous media of height $\mathrm{O}\left(\varepsilon^{2} R\right)$.

To complement the results of this study, further experimental investigation is also required to accurately determine the permeabilities of substrates formed of regular arrays of pillars, which would enable further quantitative comparisons of experimental and calculated bubble volumes. The current study is limited to porous substrates of small height and a restricted permeability range. For higher permeability substrates further analysis is required, perhaps replacing the Stokes flow in the gas film and the Darcy flow in the substrate, with a single gas domain Darcy-Brinkman formulation throughout. The Darcy-Brinkman formulation has successfully been applied to model the flow at the interface between a pure fluid and a porous media [22,29], while within the Darcy-Brinkman formulation the empirical Beavers-Joseph coefficient is also eliminated, although another empirical coefficient, the effective viscosity, is introduced. The single gas domain Darcy-Brinkman formulation may also allow the free-surface evolution to be predicted after the initial touchdown on the top surface of the substrate as it moves into the porous substrate. In any case further experimental work is required to validate either the Beavers-Joseph model or the alternative Darcy-Brinkman formulation in gas flows occurring at the small length scales of interest here.

For substrates of greater depth, two additional regimes are present, which are also worthy of further investigation. For substrate heights $\tilde{h} \gg \varepsilon^{2} R$, the analysis of the substrate flow based upon disparate horizontal and vertical length scale is no longer appropriate. In this regime the leading-order substrate pressure satisfies Laplace's equation. In two-dimensional substrates of height $\tilde{h} \sim \varepsilon R$, complex variable methods and an integration contour integration which takes in the top surface of the porous media and its impermeable base can be used to determine the infiltration velocity of gas into the substrate, which is required in the gas squeeze film equation. For substrate heights $\tilde{h} \gg \varepsilon R$, the leading-order gas cushioning is no-longer influenced by the impermeable base of the substrate. The porous substrate height $\mathrm{O}(\varepsilon R)$ marking the limit of the regime in which the impermeable base effects the leading-order gas cushioning is the same height as previously determined for a liquid layer coating an impermeable base [16]. Finally, through extensions of the analysis of Hicks and Purvis [15], three-dimensional cushioned impacts with anisotropic substrates could be investigated. As a result of variations in the two in-plane substrate effective permeabilities, situations are envisaged where splashing may be promoted in one direction and not the other.

Acknowledgements The authors are grateful to Dr. Manish Tiwari for introducing them to experiments involving droplet impacts with textured substrates. PDH is grateful for the use of the high performance computing facilities at the University of Aberdeen. RP is grateful for the use of the High Performance Computing Cluster supported by the Research and Specialist Computing Support service at the University of East Anglia. 


\section{References}

1. Alizadeh, A., Bahadur, V., Zhong, S., Shang, W., Li, R., Ruud, J., Yamada, M., Ge, L., Dhinojwala, A., Sohal, M.: Temperature dependent droplet impact dynamics on flat and textured surfaces. Appl. Phys. Lett. 100(11), 111601 (2012). DOI 10.1063/1.3692598

2. Beavers, G.S., Joseph, D.D.: Boundary conditions at a naturally permeable wall. J. Fluid Mech. 30, 197-207 (1967). DOI 10.1017/S0022112067001375

3. Bouwhuis, W., van der Veen, R.C.A., Tran, T., Keij, D.L., Winkels, K.G., Peters, I.R., van der Meer, D., Sun, C., Snoeijer, J.H., Lohse, D.: Maximal air bubble entrainment at liquid-drop impact. Phys. Rev. Lett. 109, 264501 (2012). DOI 10.1103/PhysRevLett.109.264501

4. Chandra, S., Avedisian, C.T.: On the collision of a droplet with a solid surface. Proc. Roy. Soc. Lond. A 432(1884), 13-41 (1991). DOI 10.1098/rspa.1991.0002

5. Cooker, M.J.: A theory for the impact of a wave breaking onto a permeable barrier with jet generation. J. Eng. Math. 79, 1-12 (2012). DOI 10.1007/s10665-012-9558-9

6. van Dam, D.B., Le Clerc, C.: Experimental study of the impact of an ink-jet printed droplet on a solid substrate. Phys. Fluids 16(9), 3403-3414 (2004). DOI 10.1063/1.1773551

7. Delbos, A., Lorenceau, E., Pitois, O.: Forced impregnation of a capillary tube with drop impact. J. Colloid Interface Sci. 341(1), 171-177 (2010). DOI 10.1016/j.jcis.2009.09.016

8. Dell'Aversana, P., Tontodonato, V., Carotenuto, L.: Suppression of coalescence and of wetting: The shape of the interstitial film. Phys. Fluids 9(9), 2475-2485 (1997). DOI 10.1063/1.869471

9. Ding, H., Theofanous, T.G.: The inertial regime of drop impact on an anisotropic porous substrate. J. Fluid Mech. 691, 546-567 (2012). DOI 10.1017/jfm.2011.491

10. Driscoll, M.M., Nagel, S.R.: Ultrafast interference imaging of air in splashing dynamics. Phys. Rev. Lett. 107(15), 154502 (2011). DOI 10.1103/PhysRevLett.107.154502

11. Ellis, A.S., Smith, F.T., White, A.H.: Droplet impact onto a rough surface. Q. J. Mech. Appl. Math. 64(2), 107-139 (2011). DOI 10.1093/qjmam/hbq026

12. Fitt, A.D., Howell, P.D., King, J.R., Please, C.P., Schwendeman, D.W.: Multiphase flow in a roll press nip. Eur. J. Appl. Math. 13(3), 225-259 (2002). DOI 10.1017/S0956792501004636

13. Han, D., Steckl, A.J.: Superhydrophobic and oleophobic fibers by coaxial electrospinning. Langmuir 25(16), 9454-9462 (2009). DOI 10.1021/la900660v

14. Hicks, P.D., Ermanyuk, E.V., Gavrilov, N.V., Purvis, R.: Air trapping at impact of a rigid sphere onto a liquid. J. Fluid Mech. 695, 310-320 (2012). DOI 10.1017/jfm.2012.20

15. Hicks, P.D., Purvis, R.: Air cushioning and bubble entrapment in three-dimensional droplet impacts. J. Fluid Mech. 649, 135-163 (2010). DOI 10.1017/S0022112009994009

16. Hicks, P.D., Purvis, R.: Air cushioning in droplet impacts with liquid layers and other droplets. Phys. Fluids 23(6), 062104 (2011). DOI 10.1063/1.3602505

17. Hicks, P.D., Purvis, R.: Liquid-solid impacts with compressible gas cushioning. J. Fluid Mech. 735, 120-149 (2013). DOI 10.1017/jfm.2013.487

18. Iafrati, A., Korobkin, A.A.: Self-similar solutions for porous/perforated wedge entry problem. In: Proceedings of the 20th International Workshop on Water Waves and Floating Bodies, Longyearbyen, Norway (2005)

19. Jones, I.P.: Low reynolds number flow past a porous spherical shell. Math. Proc. Cambridge Phil. Soc. 73, 231-238 (1973). DOI 10.1017/S0305004100047642

20. King, F.W.: Hilbert Transforms, Encyclopedia of Mathematics and its Applications, vol. 1. Cambridge University Press. Cambridge (2009). DOI 10.1017/CBO9780511721458

21. Knox, D.J., Wilson, S.K., Duffy, B.R., McKee, S.: Porous squeeze-film flow. IMA J. Appl. Math. 80(2), 376-409 (2015). DOI 10.1093/imamat/hxt042

22. Le Bars, M., Worster, M.G.: Interfacial conditions between a pure fluid and a porous medium: implications for binary alloy solidification. J. Fluid Mech. 550, 149-173 (2006). DOI 10.1017/ S0022112005007998

23. Lembach, A.N., Tan, H.B., Roisman, I.V., Gambaryan-Roisman, T., Zhang, Y., Tropea, C., Yarin, A.L.: Drop impact, spreading, splashing, and penetration into electrospun nanofiber mats. Langmuir 26(12), 9516-9523 (2010). DOI 10.1021/la100031d

24. Liu, Y., Moevius, L., Xu, X., Qian, T., Yeomans, J.M., Wang, Z.: Pancake bouncing on superhydrophobic surfaces. Nat. Phys. 10, 515-519 (2014). DOI 10.1038/nphys2980

25. Liu, Y., Tan, P., Xu, L.: Compressible air entrapment in high-speed drop impacts on solid surfaces. J. Fluid Mech. 716, R9 (2013). DOI 10.1017/jfm.2012.583

26. Maitra, T., Antonini, C., Tiwari, M.K., Mularczyk, A., Imeri, Z., Schoch, P., Poulikakos, D.: Supercooled water drops impacting superhydrophobic textures. Langmuir 30(36), 10,855-10,861 (2014). DOI 10. 1021/la502675a 
27. Maitra, T., Tiwari, M.K., Antonini, C., Schoch, P., Jung, S., Eberle, P., Poulikakos, D.: On the nanoengineering of superhydrophobic and impalement resistant surface textures below the freezing temperature. Nano Lett. 14(1), 172-182 (2014). DOI 10.1021/nl4037092

28. Mandre, S., Mani, M., Brenner, M.P.: Precursors to splashing of liquid droplets on a solid surface. Phys. Rev. Lett. 102(13), 134502 (2009). DOI 10.1103/PhysRevLett.102.134502

29. Nabhani, M., El Khlifi, M., Bou-saïd, B.: A numerical simulation of viscous shear effects on porous squeeze-film using the Darcy-Brinkman model. Mech. Ind. 11, 327-337 (2010). DOI 10.1051/meca/ 2010046

30. Nield, D.A.: The Beavers-Joseph boundary condition and related matters: a historical and critical note. Transp. Porous Med. 78(3), 537-540 (2009). DOI 10.1007/s11242-009-9344-y

31. Purvis, R., Smith, F.T.: Air-water interactions near droplet impact. Eur. J. Appl. Math. 15, 853-871 (2004). DOI 10.1017/S0956792504005674

32. Raza, M.A., van Swigchem, J., Jansen, H.P., Zandvliet, H.J.W., Poelsema, B., Kooij, E.S.: Droplet impact on hydrophobic surfaces with hierarchical roughness. Surf. Topogr. Metrol. Prop. 2(3), 035002 (2014). DOI 10.1088/2051-672X/2/3/035002

33. de Ruiter, J., van den Ende, D., Mugele, F.: Air cushioning in droplet impact. II. Experimental characterization of the air film evolution. Phys. Fluids 27(1), 012105 (2015). DOI http://dx.doi.org/10.1063/1. 4906115

34. Saffman, P.G.: On the boundary condition at the surface of a porous medium. Stud. Appl.Math. 50, 93-101 (1971)

35. Smith, F.T., Li, L., Wu, G.X.: Air cushioning with a lubrication/inviscid balance. J. Fluid Mech. 482, 291-318 (2003). DOI 10.1017/S0022112003004063

36. Srikar, R., Gambaryan-Roisman, T., Steffes, C., Stephan, P., Tropea, C., Yarin, A.L.: Nanofiber coating of surfaces for intensification of drop or spray impact cooling. Int. J. Heat Mass Trans. 52(25-26), 5814-5826 (2009). DOI http://dx.doi.org/10.1016/j.ijheatmasstransfer.2009.07.021

37. Thoroddsen, S.T., Etoh, T.G., Takehara, K.: Air entrapment under an impacting drop. J. Fluid Mech. 478, 125-134 (2003). DOI 10.1017/S0022112002003427

38. Thoroddsen, S.T., Etoh, T.G., Takehara, K., Ootsuka, N., Hatsuki, Y.: The air bubble entrapped under a drop impacting on a solid surface. J. Fluid Mech. 545, 203-212 (2005). DOI 10.1017/ S0022112005006919

39. Tran, T., de Maleprade, H., Sun, C., Lohse, D.: Air entrainment during impact of droplets on liquid surfaces. J. Fluid Mech. 726, R6 (2013). DOI 10.1017/jfm.2013.261

40. Tran, T., Staat, H.J.J., Susarrey-Arce, A., Foertsch, T.C., van Houselt, A., Gardeniers, H.J.G.E., Prosperetti, A., Lohse, D., Sun, C.: Droplet impact on superheated micro-structured surfaces. Soft Matter 9, 3272-3282 (2013). DOI 10.1039/C3SM27643K

41. Tsai, P., van der Veen, R.C.A., van de Raa, M., Lohse, D.: How micropatterns and air pressure affect splashing on surfaces. Langmuir 26(20), 16,090-16,095 (2010). DOI 10.1021/la102330e

42. van der Veen, R.C.A., Hendrix, M.H.W., Tran, T., Sun, C., Tsai, P.A., Lohse, D.: How microstructures affect air film dynamics prior to drop impact. Soft Matter 10, 3703-3707 (2014). DOI 10.1039/C4SM00298A

43. van der Veen, R.C.A., Tran, T., Lohse, D., Sun, C.: Direct measurements of air layer profiles under impacting droplets using high-speed color interferometry. Phys. Rev. E 85, 026315 (2012). DOI 10. 1103/PhysRevE.85.026315

44. Wilson, S.K.: A mathematical model for the initial stages of fluid impact in the presence of a cushioning fluid layer. J. Eng. Math. 25(3), 265-285 (1991). DOI 10.1007/BF00044334

\section{A Gas cushioning with alternative forms of the Beavers-Joseph boundary condition}

In addition to the full Beavers-Joseph boundary condition on the surface of a shallow porous substrate (30), two simplified versions of this expression are also considered. These correspond to no slip in the substrate ( $\delta=0)$ and the absence of velocity shear in the gas film $(\delta=0$ and $\alpha \rightarrow \infty)$. In these cases the Reynolds lubrication equation (36) simplifies to give

$$
\frac{\partial f}{\partial t}=\frac{1}{12} \frac{\partial}{\partial x}\left[\frac{f^{2}\left(\alpha f^{2}+4 k^{1 / 2} f\right)}{\alpha f+k^{1 / 2}} \frac{\partial p}{\partial x}\right]+k h \frac{\partial^{2} p}{\partial x^{2}}
$$

and

$$
\frac{\partial f}{\partial t}=\frac{1}{12} \frac{\partial}{\partial x}\left[f^{3} \frac{\partial p}{\partial x}\right]+k h \frac{\partial^{2} p}{\partial x^{2}}
$$




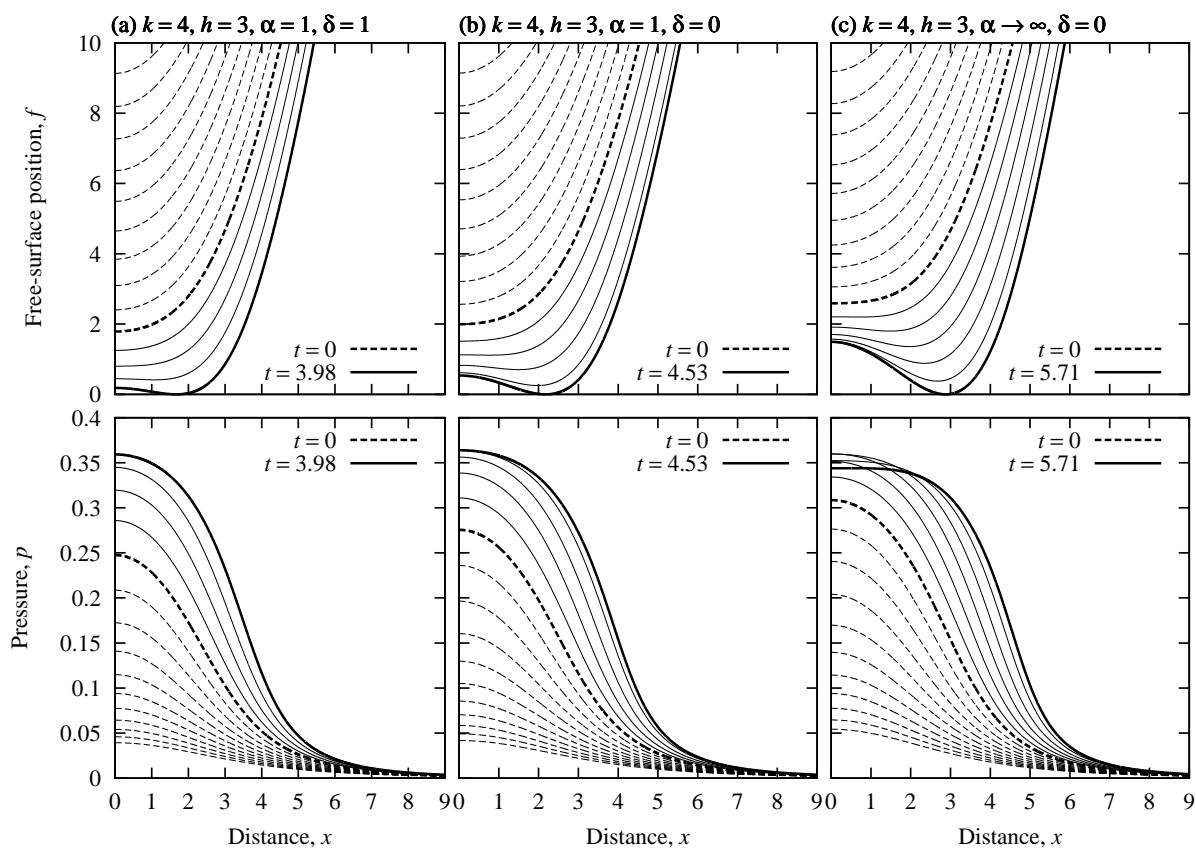

Fig. 9: Droplet free-surface and pressure evolution in gas cushioned impacts for $k=4$ and $h=3$, with simplified forms of the Beavers-Joseph boundary condition.

respectively, while the boundary integral (13) and the initial and far-field conditions remain unchanged.

Comparative results for these alternative boundary conditions are presented in figure 9 alongside the full Beavers-Joseph condition for the case $k=4$ and $h=3$. The full Beavers-Joseph condition (shown in figure 9(a)), results in a smaller pocket of trapped gas compared to the other two boundary conditions, this is because this case has the greatest amount of gas velocity slip at the substrate interface and so so the gas is more able to escape from beneath the oncoming droplet. The trapped gas pocket in figure 9(c) where $\alpha \rightarrow \infty$, $\delta=0$ is the largest gas pocket of the three, as this boundary condition corresponds to no-slip for the gas on the substrate surface and hence the gas is less able to escape from underneath the oncoming droplet. The variations between the different forms of the boundary condition diminish as the value of $k$ falls. In cases where the substrate permeability is well known, a detailed comparison of the radius of the air pocket both experimentally and using the models described herein, would enable an accurate determination of the parameters in the Beavers-Joseph condition.

An extension to the Beavers-Joseph boundary condition (8) due to Jones [19]

$$
\frac{K^{1 / 2}}{\alpha}\left(\frac{\partial \tilde{u}_{g}}{\partial \tilde{y}}+\frac{\partial \tilde{v}_{g}}{\partial \tilde{x}}\right)=\tilde{u}_{g}-\delta \tilde{u}_{s}
$$

is often applied to non-parallel flows where (as herein) $\tilde{v}(x, 0, t) \neq 0$. This formula, which has never been explicitly verified experimentally [30], includes shear stress instead of just velocity shear. However, because of the disparate length and velocity scales (15-16) in the gas film, the second term on the left-hand side is $\mathrm{O}\left(\varepsilon^{2}\right)$ smaller than the first term. Consequently if this extra term was included in the analysis, then it would be neglected at leading order leaving the Beavers-Joseph condition (8) as used. 


\section{B Numerical solution of the free-surface integral with surface tension}

The gas pressure and the deviation of the droplet free-surface from its undisturbed position are expressed as complex Fourier series

$$
\begin{array}{r}
p_{g}(x, t)=\sum_{n=-\infty}^{\infty} P_{n}(t) \exp \left(\frac{\mathrm{i} n \pi x}{L}\right), \\
f(x, t)-\frac{x^{2}}{2}+t=\sum_{n=-\infty}^{\infty} F_{n}(t) \exp \left(\frac{\mathrm{i} n \pi x}{L}\right),
\end{array}
$$

where the Fourier coefficients are given by

$$
\begin{aligned}
& P_{n}(t)=\frac{1}{2 L} \int_{-L}^{L} p_{g}(x, t) \exp \left(-\frac{\mathrm{i} n \pi x}{L}\right) \mathrm{d} x, \\
& F_{n}(t)=\frac{1}{2 L} \int_{-L}^{L}\left(f(x, t)-\frac{x^{2}}{2}+t\right) \exp \left(-\frac{\mathrm{i} n \pi x}{L}\right) \mathrm{d} x .
\end{aligned}
$$

Here the droplet free-surface repeats with period $2 L$, although by choosing a spatial domain $-L<x<L$ and $L$ suitably large, edge effects are negligible. The deviation of the droplet free-surface from its undisturbed position is approximated by the Fourier series rather than the free-surface position itself in order to increase the smoothness of the Fourier series at $x= \pm L$.

The integral

$$
\mathscr{H}[g(x)] \equiv \frac{1}{\pi} f_{-\infty}^{\infty} \frac{g(\xi) \mathrm{d} \xi}{x-\xi},
$$

is the Hilbert transform of some function $g(x)$. Consequently upon substituting the Fourier series (57) into the integral equation (48)

$$
\begin{aligned}
\sum_{n=-\infty}^{\infty} \frac{\mathrm{d}^{2} F_{n}}{\mathrm{~d} t^{2}}(t) \exp \left(\frac{\mathrm{i} n \pi x}{L}\right)= & \sum_{n=-\infty}^{\infty} \frac{\mathrm{i} n \pi}{L} P_{n}(t) \mathscr{H}\left[\exp \left(\frac{\mathrm{i} n \pi x}{L}\right)\right] \\
& +\sigma \sum_{n=-\infty}^{\infty}-\frac{\mathrm{i} n^{3} \pi^{3}}{L^{3}} F_{n}(t) \mathscr{H}\left[\exp \left(\frac{\mathrm{i} n \pi x}{L}\right)\right] .
\end{aligned}
$$

Using properties of the Hilbert transform [20]

$$
\mathscr{H}\left[\exp \left(\frac{\mathrm{i} n \pi x}{L}\right)\right]=-\mathrm{i} \operatorname{sgn}(n) \exp \left(\frac{\mathrm{i} n \pi x}{L}\right),
$$

and hence upon collecting coefficients of matching exponential terms, the Fourier coefficients are related through

$$
\frac{\mathrm{d}^{2} F_{n}}{\mathrm{~d} t^{2}}(t)+\sigma \frac{|n|^{3} \pi^{3}}{L^{3}} F_{n}(t)=\frac{|n| \pi}{L} P_{n}(t) .
$$

The Fourier series (57) are truncated and sufficient terms are retained to achieve convergence. Given a pressure profile $p_{g}(x, t)$, the Fourier pressure coefficients are obtained by means of a Fast Fourier Transform. Subsequently (61) can be discretized in time and used to calculate the evolution the corresponding freesurface Fourier coefficients, which (using an inverse Fast Fourier Transform), allow the free-surface profile to be recovered. 